\title{
Measurement of Self-emitting Magnetic Signals from a Precut Notch of Q235 Steel during Tensile Test
}

\author{
Lihong Dong, Binshi Xu, and Shiyun Dong \\ National Key Laboratory for Remanufacturing, Academy of Armored Forces Engineering, \\ Beijing, 100072, P.R. China \\ linghong. dong@126.com
}

\begin{abstract}
In order to investigate the approach to characterize the damage behavior of ferromagnetic material components with flaw by metal magnetic memory testing, a sheet specimen with a precut notch of Q235 steel was chosen to measure the self-emitting magnetic signals on its surface during tensile test. The results showed that an abnormal magnetic signal appeared at the position of the precut notch after early loading and then transformed gradually to a clear magnetic abnormal peak with the tensile load increasing, which implied that the variations of self-emitting signals can reflect the influence of applied load on the specimen dynamically. The above-mentioned phenomenon was discussed from the point of view of magnetomechanical effect.
\end{abstract}

Keywords: self-emitting signals, applied load, damage, magnetic abnormal peak, effective magnetic field.

\section{Introduction}

Ferromagnetic parts are widely used in engineering fields [1-3]. During their manufacturing process or operation, various defects will unavoidably occur in the materials [4-6]. In most cases the ferromagnetic components have to work with these defects. Hence, monitoring the changes of the defects dynamically in service is very important to ensure the safety of the components.

However, most of the available non-destructive testing methods, such as ultrasonic inspection, eddy current inspection, penetrant inspection, and magnetic particle inspection, can only examine macroscopic defect statically, which are not suitable for monitoring microscopic defect change dynamically[7]. At present acoustic emission testing is the only dynamic method for early damage diagnosis. However, the measured components must be loaded by using acoustic emission testing, and it is difficult to analyze the acoustic emission signals due to existing noise interference, which limits the method practical application[8,9].

Metal Magnetic Memory testing (MMMT) is a relatively new non-destructive testing method which was first put forward by Russian researchers[10-12]. The method is regarded as another non-destructive technique for early damage diagnosis, which can find the stress concentration zone of ferromagnetic materials. However, due to lack of fundamental investigation, it is still a problem how to characterize the damage behavior of ferromagnetic materials by using metal magnetic memory signals[13]. 
In this paper a sheet specimen of Q235 steel with a precut surface notch was chosen to find the mode of metal magnetic memory method characterizing damage behaviour by means of tensile test.

\section{Experiment}

\subsection{Specimen Preparation}

The test material is Q235 steel which is widely used in different engineering fields. Its chemical composition and mechanical properties can be found in references [14].

The geometry and dimension of specimen are shown in Fig.1. A precut notch, which length is $10 \mathrm{~mm}$, width is $0.5 \mathrm{~mm}$, depth is $0.5 \mathrm{~mm}$, was machined on the center of the specimen surface by electric discharge machining technique. Three measured lines of $90 \mathrm{~mm}$ length with an equal interval were arranged through the notch on the surface of the specimen.

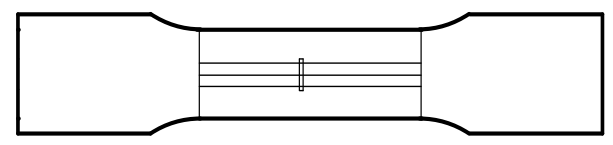

Fig. 1. The precut notch and measured lines on the specimen

\subsection{Experimental Set-Up}

The tensile test was carried out with a servo hydraulic MTS810 testing machine with static error $\pm 0.5 \%$. The metal magnetic memory signals, $H_{p}(y)$ signals (i.e. the component of self-leakage field), were measured by an EMS-2003 Metal Magnetic Memory instrument. In the tensile test, the specimen was vertically held between the upper and lower grip holders of the testing machine. When loaded to a predetermined load and retained some time, the specimen was taken carefully from the machine and placed on a non-magnetic electrical scanning platform along the south-north axis. Then, the $\mathrm{H}_{\mathrm{p}}(\mathrm{y})$ values of all three measured lines were collected. After measurement, the specimen was loaded again to a higher predetermined stress, and the above measuring procedure was repeated until the specimen failed.

\section{Results and Discussion}

\subsection{Distributions of $H_{p}(y)$ Signals during the Test}

During the test, only elastic deformation occurred in the specimen if the applied load was less than $40 \mathrm{kN}$. The specimen yielded when the tensile load was up to $49 \mathrm{kN}$, and at last it was failed at $68 \mathrm{kN}$. The distributions of $\mathrm{H}_{\mathrm{p}}(\mathrm{y})$ signals at initial state before loading, $1 \mathrm{kN}, 10 \mathrm{kN}, 40 \mathrm{kN}, 60 \mathrm{kN}$ and $65 \mathrm{kN}$ respectively were shown in Fig. 2 . 


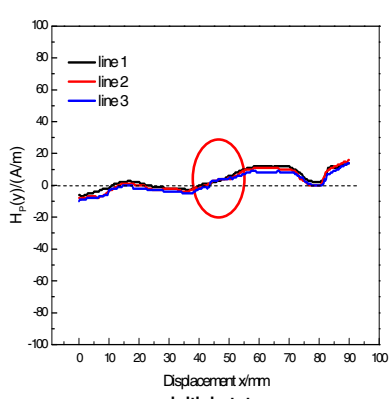

initial state

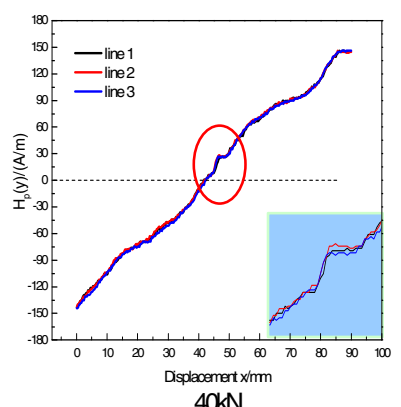

$40 \mathrm{kN}$

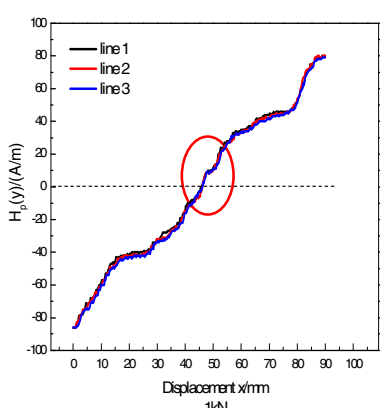

$1 \mathrm{kN}$

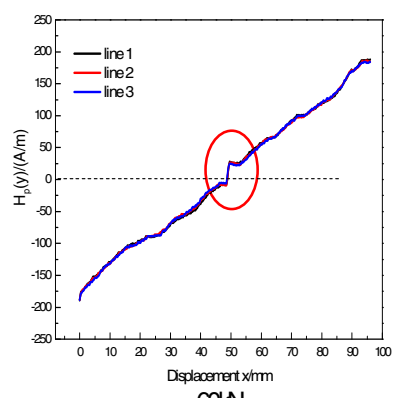

$60 \% N$

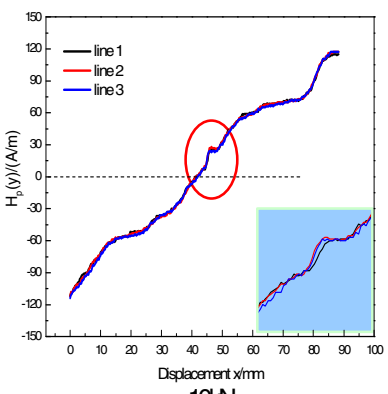

$10 \mathrm{KN}$

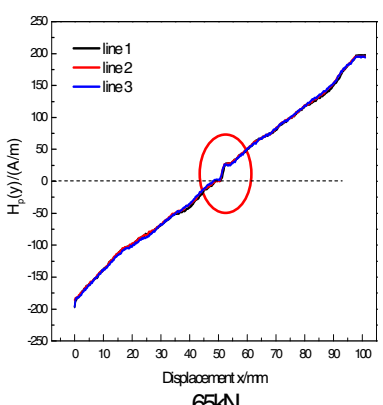

$65 \mathrm{kN}$

Fig. 2. The distribution of $\mathrm{H}_{\mathrm{p}}(\mathrm{y})$ signals on the three measured lines during tensile test

In Fig.2 the red circle indicated the position of the precut notch. Because the specimen was demagnetized before tensile test, the value of initial magnetic signals were very small, which were in the range of $\pm 20 \mathrm{~A} / \mathrm{m}$.

The initial $\mathrm{H}_{\mathrm{p}}(\mathrm{y})$ curve of the specimen had a curly feature to some degree, no abnormal magnetic signals were observed on the position of the precut notch.

After a tensile load of $1 \mathrm{kN}$ was applied to the specimen, the magnetic signal curves on the three measured lines presented a feature of magnetic ordering, which left signals were negative and right signals were positive. At that time a very weak abnormal signal was observed at the position of the precut notch, which was induced by the load of $1 \mathrm{kN}$. If the position of the precut notch was not known beforehand, the abnormal signal might be ignored. The result showed that the applied load can excite the precut notch to generate self-emitting signals and change the magnetism of the specimen. However, the load of $1 \mathrm{kN}$ was too small to produce a strong feature of abnormal magnetic signals.

When loaded up to $10 \mathrm{kN}$, a rough abnormal magnetic peak appeared, indicating the existence of the precut notch. At $40 \mathrm{kN}$, the shape of the abnormal magnetic peak became clearer continuously. When the applied load increased up to $60 \mathrm{kN}$, the magnetic signal curve became an approximate sloping straight line, and the abnormal magnetic peak at the position of the precut notch showed a clear feature of crest and trough. The last figure indicated the magnetic state on the measured lines before failure. At that time the applied load is $65 \mathrm{kN}$, and the specimen had a high elongation. 
Moreover, observed by a reading microscope, it was found that the width of the precut notch increased whereas the depth of the notch decreased, leading to the abnormal magnetic signals weakening.

The above results showed that a applied load can change the magnetism of ferromagnetic materials. With the applied load increasing, the magnetism increased. Hence the precut notch emitted magnetic signals which presented a feature of abnormal magnetic peak. Moreover, the distributions of the abnormal magnetic peak changed with the applied load increasing. Hence it can be inferred that metal magnetic memory testing has the ability to monitor the variations of defect in ferromagnetic materials dynamically. During measurement ferromagnetic materials need not loaded. The feature signals of defect are clear enough to further analyze.

\subsection{Load-Induced Magnetism Enhancement}

The above mentioned results showed that an applied load on the specimen, even if very small, could change the magnetism of the tested ferromagnetic material under the Earth magnetic field. Thus the applied load can be seen as a external excitation, which cause the abnormal magnetic signals occurring and enhancing at the precut notch.

According to the magnetomechanical effect, when an axial tensile load is applied to ferromagnetic specimen, the specimen will deform, leading to the appearance of stress energy of $E_{\sigma}[15]$ :

$$
E_{\sigma}=-\frac{3}{2} \sigma \lambda\left(\cos ^{2} \theta-v \sin ^{2} \theta\right)
$$

Consequently, the applied load alter the magnetism of the ferromagnetic specimen, causing an effect magnetic field $\mathrm{H}_{\sigma}$ on the specimen:

$$
H_{\sigma}=\frac{1}{\mu_{0}} \frac{\partial E_{\sigma}}{\partial M}=\frac{3}{2} \frac{\sigma}{\mu_{0}}\left(\frac{\partial \lambda}{\partial M}\right)_{\sigma}\left(\cos ^{2} \theta-v \sin ^{2} \theta\right)
$$

Under the effect of $\mathrm{H}_{\sigma}$, on the one hand, the magnetic charges will accumulate on both ends of the specimen, causing different polarities, $\mathrm{N}$ and $\mathrm{S}$, appearing at the two ends. Hence the magnetic signal curve exhibited good linearity after loading, which the left signals were negative while the right signals were positive. With the axial load increasing the effect magnetic field $\mathrm{H}_{\sigma}$ increased, thus the magnetism of the specimen increased too, leading to the gradual disappear of the curl of the magnetic signal curve and the increase of the linearity and the slope coefficient of magnetic signal curve.

On the other hand, when the specimen was magnetized by the effect magnetic field, the precut notch on the surface cut the path of magnetic lines of $\mathrm{H}_{\sigma}$. Similarly, the magnetic charges with different polarities accumulated on both sides of the notch. Consequently, the abnormal magnetic peak gradually appeared with the load increasing. Consequently, the amplitude of load applied to ferromagnetic materials and their deformation can be inferred by measuring and analyzing the feature signals of defects, thus the damage degree of ferromagnetic components can be known in service. The schematic diagram was shown in Fig.3. 


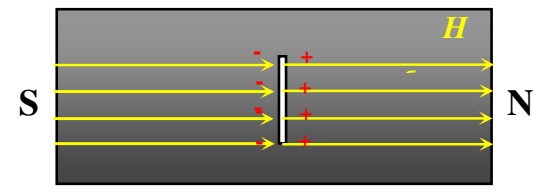

Fig. 3. The generation of magnetic polarities induced by $\mathrm{H}_{\sigma}$ on the surface of the specimen

\section{Conclusions}

When a axial tensile load was applied to the ferromagnetic specimen, an effective magnetic field was occurred, causing opposite magnetic charges accumulation on the both ends of the precut notch. Hence, the precut notch emitted abnormal magnetic signals spontaneously due to the excitation of the effective magnetic field. The abnormal magnetic peak gradually appeared with the applied load increasing, which indicated the position and amplitude of the precut defect. It is feasible to dynamically monitor the damage degree of ferromagnetic materials with defects by means of metal magnetic memory testing.

\section{References}

1. Pešička, J., Kužel, R., Dronhofer, A., Eggeler, G.: The evolution of dislocation density during heat treatment and creep of tempered martensite ferrite steels. Acta Materialia 51, 4847-4862 (2003)

2. Hu, J.-f., Yang, J.-g., Fang, H.-y., Li, G.-m., et al.: Temperature, stress and microstructure in $10 \mathrm{Ni} 5 \mathrm{CrMoV}$ steel plate during air-arc cutting process. Computational Materials Science 38(4), 631-641 (2007)

3. Colombi, P., Poggi, C.: Strengthening of tensile steel members and bolted joints using adhesively bonded CFRP plates. Construction and Building Materials 20(1-2), 22-33 (2007)

4. Gao, N., Dwyer-Joyce, R.S., Beynon, J.H.: Effects of surface defects on rolling contact fatigue of 60/40 brass. Wear, 225-229, 983-994 (1999)

5. El-Batahgy, A., Zaghloul, B.: Fatigue failure of an offshore condensate recycle line in a natural gas production field. Materials Characterization 54(3), 246-253 (2005)

6. Kuźnicka, B., Stróżyk, P.: Failure analysis of disintegrator beaters. Engineering Failure Analysis 13(1), 155-162 (2006)

7. Li, J.-w., Chen, J.-m.: Non-destructive testing handbook. Mechanical Engineering Publisher, Beijing (2002) (in Chinese)

8. Ken, W., Junichiro, N., Mitsuyasu, I., Hiroshi, Y.: Localized failure of concrete in compression identified by AE method. Construction and Building Materials 18(3), 189-196 (2004)

9. Al-Ghamd, A.M., Mba, D.: A comparative experimental study on the use of acoustic emission and vibration analysis for bearing defect identification and estimation of defect size. Mechanical Systems and Signal Processing 20(7), 1537-1571 (2006)

10. Doubov, A.A.: A study of metal properties using the method of magnetic memory. Metal Science and Heat Treatment 39(9-10), 401-402 (1997)

11. Doubov, A.A.: Screening of weld quality using the magnetic metal memory effect. Welding in the world 41, 196-198 (1998) 
12. Doubov, A.A.: A Technique for Monitoring the Bends of Boiler and Steam-Line Tubes Using the Magnetic Memory of Metal. Thermal Engineering 48(4), 289-295 (2001)

13. Dong, L., Xu, B., Dong, S., et al.: Investigation of metal magnetic memory signals from the surface of low-carbon steel and low-carbon alloyed steel. J. Cent. South Univ. Technol. 14(1), 24-27 (2007)

14. Dong, L., Xu, B., Dong, S., et al.: Variation of stress-induced magnetic signals during tensile testing of ferromagnetic steels. NDT\&E International 41, 184-189 (2008)

15. Naus, H.W.L.: Ferromagnetic Hysteresis and the Effective Field. IEEE Transactions on Magnetics 38(5), 3417-3419 (2002) 